\title{
Research Paper \\ The Effect of Benson Relaxation on Physiological Criteria in Patients Undergoing Coronary Artery Bypass Graft Surgery
}

\author{
Fatemeh Teimori ${ }^{1} \oplus$, Seyed Amirhossein Pishgooie ${ }^{2} \odot$, Mehdi Malmir $^{3},{ }^{*}$ Nahid Rajai $^{4} \oplus$
}

1. Instructor, PhD. Student of Health in Emergencies and Disaster, Department of Psychiatric Nursing, Nursing Faculty, Aja University of Medical Sciences, Tehran, Iran. 2. Associate Professor, PhD. Intensive Care Nursing Department, Nursing Faculty, Aja University of Medical Sciences, Tehran, Iran.

3. MSc. Nezaja 502 Army Hospital, Tehran, Iran.

4. Instructor, MSc. Department of Maternal-Infant Health, Nursing Faculty, Aja University of Medical Sciences, Tehran, Iran.

\begin{tabular}{ll}
$\begin{array}{c}\text { Use yourdevice to scan } \\
\text { and read the article online }\end{array}$ & $\begin{array}{l}\text { Cftation: Teimori F, Pishgooie S, Malmir M, Rajai N. [The Effect of Benson Relaxation on the Physiological Symptoms } \\
\text { in Patients undergoing Open Heart Surgery (Persian)]. Complementary Medicine Journal. 2019; 9(3):3812-3823. https://doi. } \\
\text { org/10.32598/cmja.9.3.3812 } \\
\text { doi https://doi.org/10.32598/cmja.9.3.3812 }\end{array}$ \\
\hline
\end{tabular}

\section{(1) (3)}

Article Info:

Received: 06 May 2019 Accepted: 21 Aug 2019 Available Online: 01 Nov 2019

Key words:

Benson relaxation, Patient, Open heart surgery, Physiological variables.

\section{A B STRACT}

Objective One of the common problems of patients undergoing open heart surgery is physiological disorders due to anxiety that can affect the result of surgery. The aim of this study was to investigate the effect of Benson relaxation on the physiological sysmptoms of patients undergoing open heart surgery. Methods This is a clinical trial performed on 90 candidates for open heart surgery referred to the hospitals affiliated to AJA University of Medical Sciences in Tehran in 2014. The subjects were randomly divided into two experimental and control groups. In both groups, the physiological symptoms (respiratory rate, pulse rate, systolic and diastolic blood pressure) of the patients were measured one day before surgery. Benson relaxation was performed on patients in the experimental group in the morning of the surgery day and the physiological symptoms of both groups were evaluated after intervention. Data analysis was performed in SPSS V. 21 software using Chi-square test, paired t-test and independent t-test at a significance level of $\mathrm{P}<0.05$.

Results There was no significant difference in physiological symptoms of both groups before intervention. After intervention, a significant difference between the groups was reported in terms of all physiological variables $(\mathrm{P}=0.001)$. Paired t-test results also showed a significant difference between the two groups before and after the intervention in terms of all physiological variables except diastolic blood pressure. Conclusion Benson relaxation significantly improved the physiological status of patients undergoing open heart surgery. It is recommended that nurses use this method to improve the physiological status of these patients.

\section{Extended Abstract}

\section{Introduction}

ardiovascular disease is one of the leading causes of death, disability, and a decline in quality of life in the world, accounting for $50 \%$ of all deaths in developing countries. Open heart surgery is one of the most important methods in treatment of heart diseases associated with a decrease in patient mortality. In developing countries, the prevalence of cardiac surgery is 860 per one million and in developed countries it is 60 per one mil-

\section{* Corresponding Author:}

Nahid Rajai, MSc.

Address: Department of Maternal-Infant Health, Nursing Faculty, Aja University of Medical Sciences, Tehran, Iran.

Tel: +98 (21) 77500404

E-mail: n.rajai22@yahoo.com 
lion. It is estimated that $93 \%$ of people who need cardiac surgery live in developing countries.

Preoperative anxiety before Coronary Artery Bypass Grafting (CABG) surgery is a risk factor for postoperative mortality. One of the important responsibilities of the nurses in the preoperative period is to maximize the physiological and mental health of the patient and help him/her to adapt to the current situation. In this regard, the tendency to use complementary medicine has increased because of its low cost, ease of use, and being uncomplicated.

Benson relaxation is one of the techniques of complementary medicine which is based on relaxation as a key element of meditation where four movements increase relaxation. Benson relaxation method affects the physiological symptoms of patients candidate for open heart surgery, especially in a military population.

\section{Methods}

This study is a clinical trial. The study population consisted of all men and women who were candidates for CABG surgery in one of the hospitals affiliated to AJA University of Medical Sciences in 2014. Considering 0.05 type I error and $80 \%$ test power, the sample size was calculated as 42 for each group. Given 10\% sample drop, the sample size was determined 45 for each group (total $=90$ ). Samples were selected by convenience sampling technqiue and randomly assigned into two groups of test and control. Written informed consent was obtained from the particpants. Data collection tools included a demographic form and a form assessing vital signs (pulse rate, systolic blood pressure rate, diastolic blood pressure rate, and respiratory rate).

Particpants completed demographic form, and their vital signs were recorded in the evening before surgery. Then, Benson relaxation technique was taught to them. The patient repeated this intervention twice before surgery (the evening before surgery and the morning of surgery) in the presence of the researcher. The relaxation music was played for further influence. The control group did not receive any intervention. The second phase of patient evaluation (vital signs checking) was peformed in the morning of surgery, $30 \mathrm{~min}-$ utes before surgery and after intervention.

The Mean \pm SD of age was $54.73 \pm 25.9$ years in the experimental group and $56.91 \pm 5.90$ years in control group. The results of the independent t-test showed no significant difference between the groups in terms of age $(\mathrm{P}=0.18)$. There was no significant difference between groups in terms of other demographic variables. Independent t-test results showed no significant difference between the groups in terms of physiological symptoms before the intervention, but there was a significant difference in all symptoms after the intervention (Table 1). Paired t-test results reported a significant difference between the two groups before and af-

Table 1. Comparing the mean of hemodynamic variables in both study groups after the intervention

\begin{tabular}{|c|c|c|c|}
\hline \multirow{2}{*}{ Variables } & \multicolumn{2}{|c|}{ Mean $\pm S D$} & \multirow{2}{*}{ Independent t-test Result } \\
\hline & Test & Control & \\
\hline \multirow{3}{*}{ Pulse rate } & \multirow{3}{*}{$72.11 \pm 27.29$} & \multirow{3}{*}{$88.13 \pm 64.19$} & $t=-6.326$ \\
\hline & & & $\mathrm{df}=88$ \\
\hline & & & $P=0.001$ \\
\hline \multirow{3}{*}{ Resporatory rate } & \multirow{3}{*}{$18.1 \pm 84.73$} & \multirow{3}{*}{$20.2 \pm 02.11$} & $t=-10.251$ \\
\hline & & & $\mathrm{df}=88$ \\
\hline & & & $P=0.001$ \\
\hline \multirow{3}{*}{ Systolic blood pressure } & \multirow{3}{*}{$124.15 \pm 78.77$} & \multirow{3}{*}{$138.20 \pm 78.23$} & $t=-3.661$ \\
\hline & & & $\mathrm{df}=88$ \\
\hline & & & $P=0.001$ \\
\hline \multirow{3}{*}{ Diastolic blood pressure } & \multirow{3}{*}{$77.10 \pm 89.25$} & \multirow{3}{*}{$82.9 \pm 11.13$} & $t=-2.062$ \\
\hline & & & $\mathrm{df}=88$ \\
\hline & & & $P=0.042$ \\
\hline
\end{tabular}


Table 2. Paired t-test results from comparing hemodynamic variables of participants before and after the intervention

\begin{tabular}{ccc}
\hline Hemodynamic Criteria & Experimental Group & Control Group \\
\hline Pulse rate & $\mathrm{t}=6.757$ & $\mathrm{t}=-11.104$ \\
& $\mathrm{df}=44$ & $\mathrm{df}=44$ \\
& $\mathrm{P}=0.001$ & $\mathrm{P}=0.001$ \\
Resporatory rate & $\mathrm{t}=12.069$ & $\mathrm{t}=-12.96$ \\
& $\mathrm{df}=44$ & $\mathrm{df}=44$ \\
& $\mathrm{P}=0.001$ & $\mathrm{P}=0.001$ \\
Systolic blood pressure & $\mathrm{t}=11.122$ & $\mathrm{t}=-2.878$ \\
& $\mathrm{df}=44$ & $\mathrm{df}=44$ \\
& $\mathrm{P}=0.001$ & $\mathrm{P}=0.006$ \\
& $\mathrm{t}=1.331$ & $\mathrm{t}=-0.025$ \\
& $\mathrm{df}=44$ & $\mathrm{df}=44$ \\
& $\mathrm{P}=0.190$ & $\mathrm{P}=0.605$ \\
\hline
\end{tabular}

ter the intervention in terms of physiological variables except for diastolic blood pressure (Table 2).

This study was conducted to evaluate the effect of Benson relaxation technqiue on the physiological symptoms of patients candidates for CABG surgery. According to the results of this study, Benson relaxation had a significant positive effect on decreasing the physiological parameters that make patients relaxed. In fact, Benson relaxation creats a balance between the anterior and posterior parts of the hypothalamus, decreases sympathetic system activity, releases catecholamines, reduces muscle spasms, and cardiac activity, and regulates breathing. Slower breathing patterns have a beneficial effect on heart rate and respiratory sinus arrhythmia, due to a relaxation that reduces sympathetic system activity and increases parasympathetic activity by decreasing metabolic energy requirement and autonomic system balance.

Considering the importance of relaxation in patients undergoing $\mathrm{CABG}$ surgery, and given that the use of complementary therapies can affects patients without complications, it is recommended that this technique should be used to relax patients undergoing CABG surgery and control their physiological conditions.
One of the limitations of this study was the failure to blind the researcher to the results of study groups because the researcher had undergone the Benson relaxation training and could not allow other researcher to perform sampling; hence, it is recommended to consider this limitation in future studies. In our study, only the one who was responsinle for the statistical analysis was blind to the groups. Future studies are suggested to evaluate the effect of this intervention on the stress and physiological symptoms of patients after CABG surgery.

\section{Ethical Considerations}

\section{Compliance with ethical guidelines}

This study was approved by the Research Ethics Committee of AJA University of Medical Sciences (code: 9319). It is also a registered clinical trial ( code: IRCT2014121520328N1). In this study, written informed consent was obtained from patients. Ethical Principles were according to The Declaration of Helsinki .

\section{Funding}

This paper was extracted from a research proposal approved by Aja University of Medical Sciences (Code: 
592163). The study received financial support from this university.

Authors' contributions

Conceptualization by Fatemeh Teimori, Seyed Amirhossein Pishgooie, and Mehdi Malmir; methodology by Fatemeh Teimori and Seyed Amirhossein Pishgooie; investigation: Fatemeh Teimori, Mehdi Malmir; Writing-original draft by Nahid Rajai; Writing-review \& editing by all authors; supervision by Fatemeh Teimori.

Conflicts of interest

The authors declared no conflict of interests.

Acknowledgements

The authors would like to thank the Aja University of Medical Sciences for their support. 
This Page Intentionally Left Blank 


\title{
بررسى تأثير آرامسازى بنسون بر معيارهاى فيزيولوزيك بيماران كانديد عمل جراحى قلب باز
}

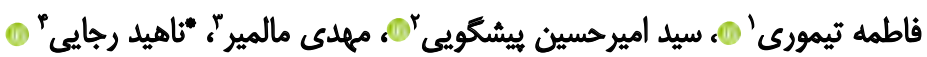

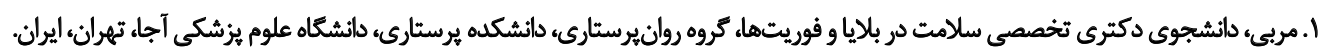

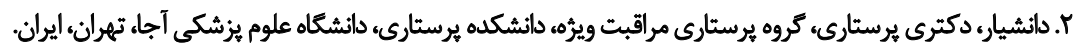

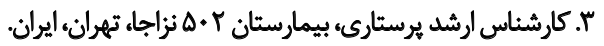

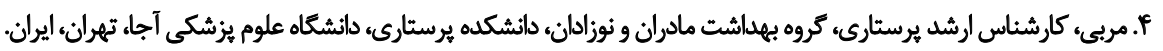

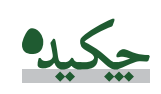

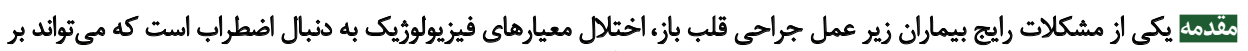

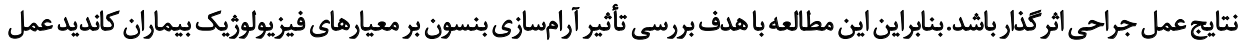
: جراحى قلب باز انجام شد.

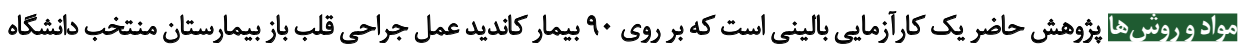

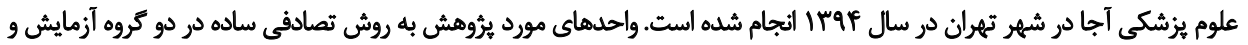

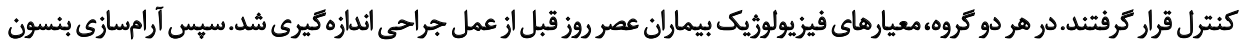

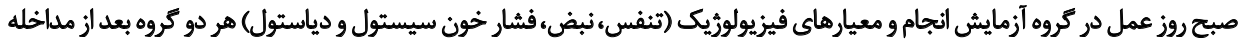

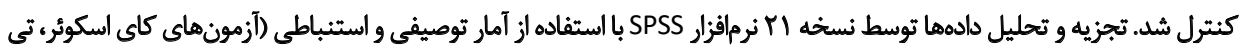

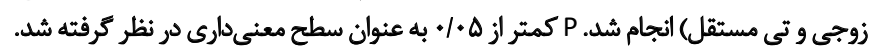

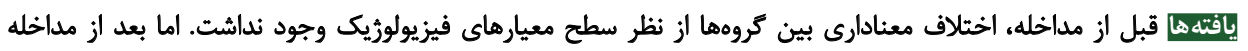

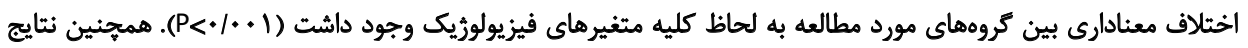

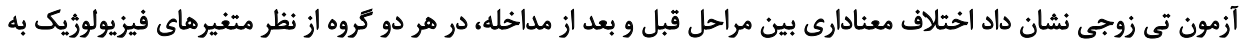
جز فشار خون دياستول وجود داشت نوان.

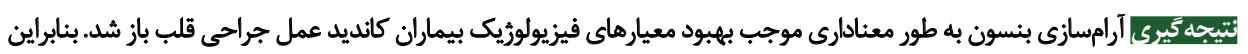

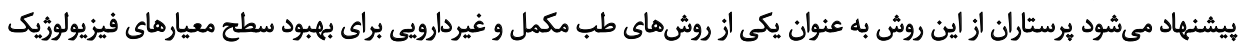

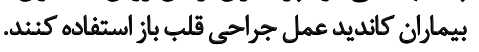

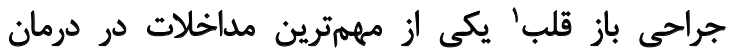

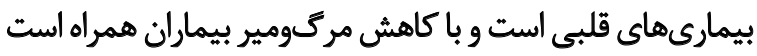

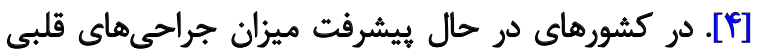

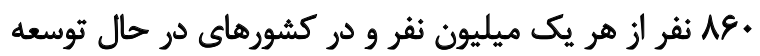

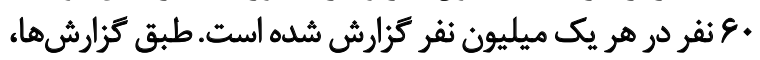

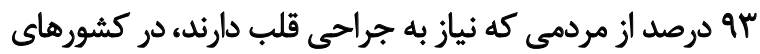

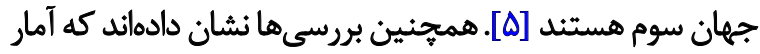

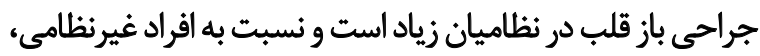

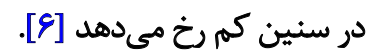
قبل از عمل جراحى باز قلب، كمبود اطلاعات، نبود حمايت
اطلاعات مقاله:

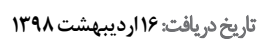

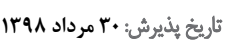

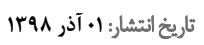

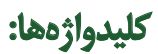

آرامسازي بئسون، بيمار، جراحي قاخ بلب بازء، شاخص هاى فيزيولوريك باري

1. Coronary Artery Bypass Grafting (CABG)

* نويسئده مسئول:

ناهيد رجائى

نشائي: تهران، دانشكاه علوم يزشكى آجا، دانشكده يرستارى، كروه بهيداشت مادران و ونوزادان.

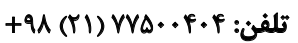
n.rajai22@yahoo.com يست الكترونيكى: 


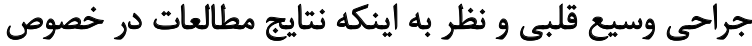

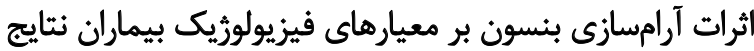

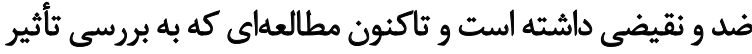

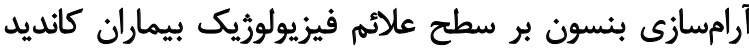

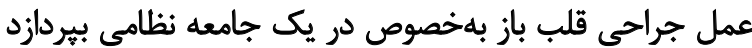

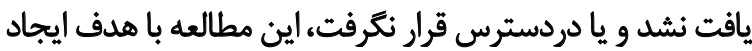

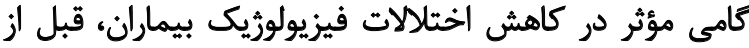

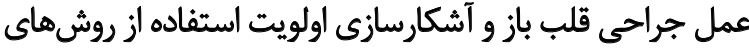

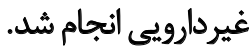

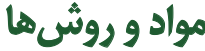

اين مطالعه يك كارآزمايى بالينى است. يّ از تصويب طرح

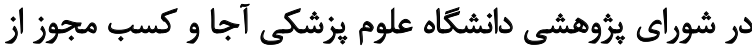

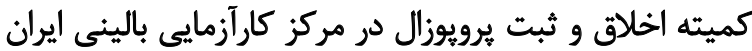

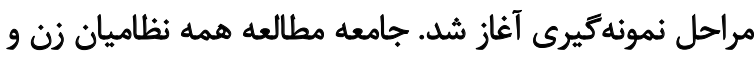

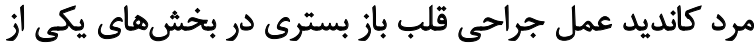

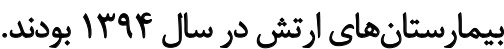
حجم نمونه از طريق فرمول يوكاك با توجه به مقاله حضرتى

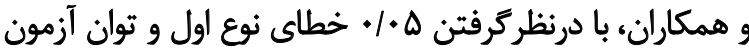

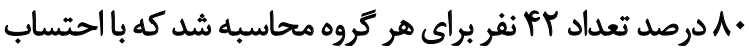

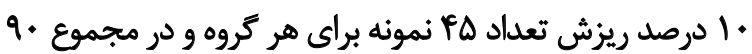

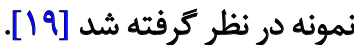

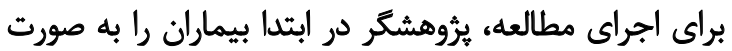

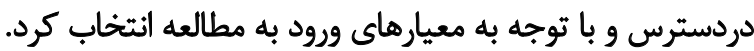

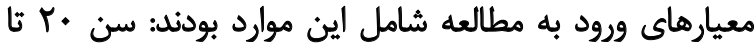

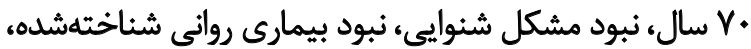

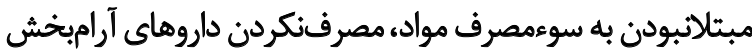

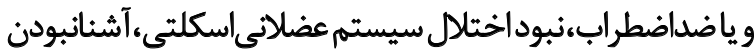

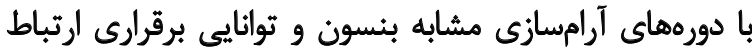

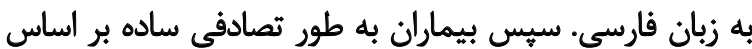

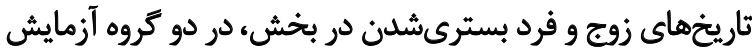

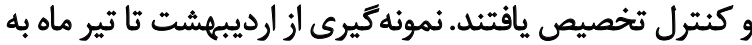
مدت سه ماه انجام شد.

قبل از شروع مطالعه اهداف يثروهش براى نمونهها توضيح داده

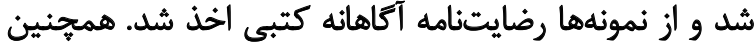

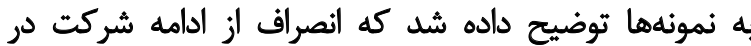

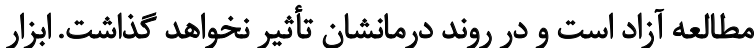

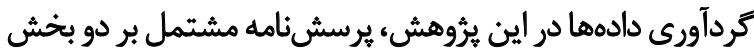

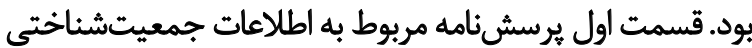

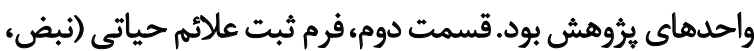

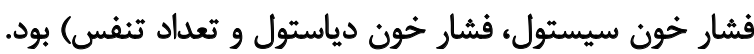

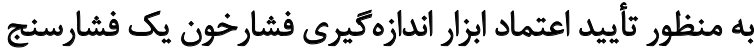

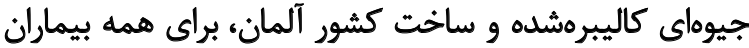

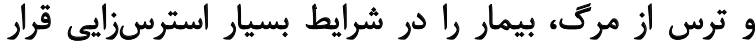

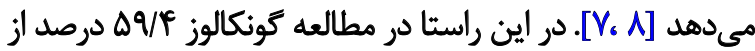

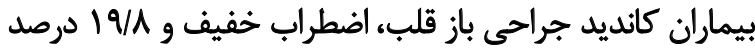

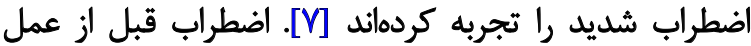

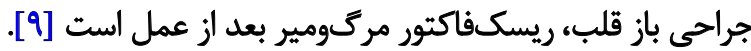

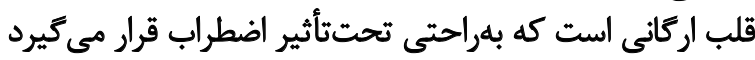

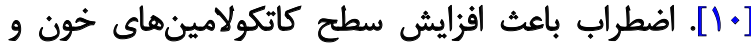

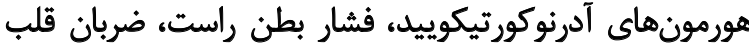

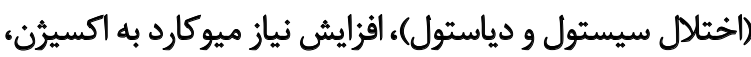

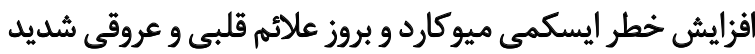

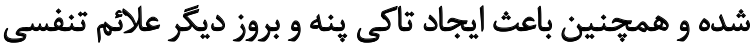

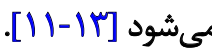
از جمله مسئوليتهاي مهمه يرستاران در دوره قبل از جراحى،

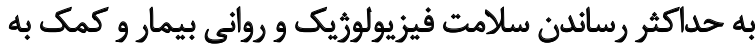

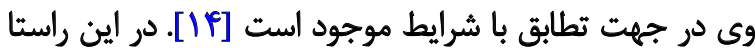

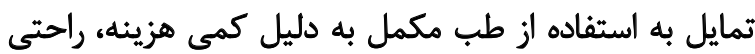

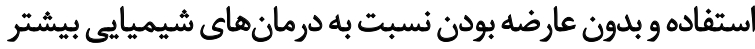

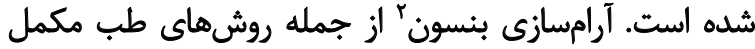

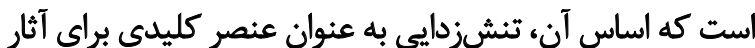

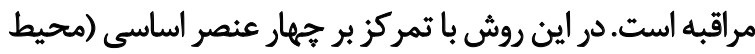

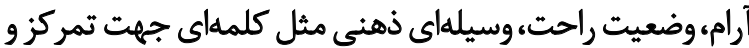

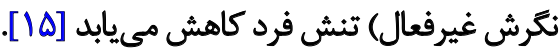
در آرامسازى بنسون عضلات شل شده، در نتيجه تعداد نبض،

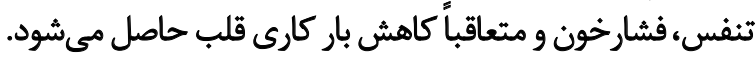

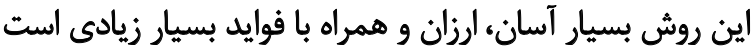

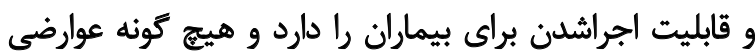

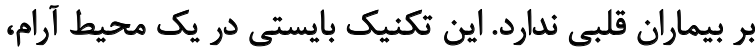

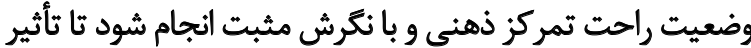

واقعى خود را بكذارد [19].

مطالعات متعددى در راستاى تأثير آرامسازى بنسون بر سطح ماته

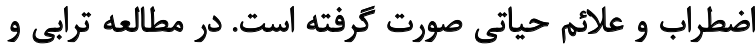

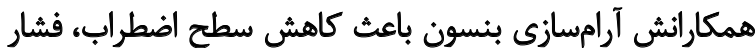

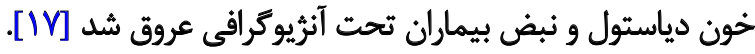

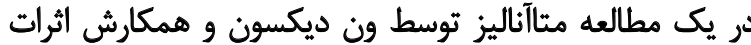

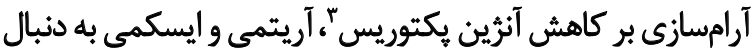

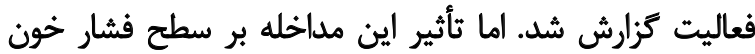

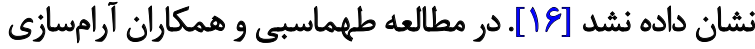

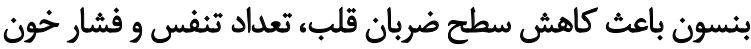
سيستول شد، اما بر فشار دياستول تأثيرى نداشت [1/11]. با توجه به نقش حياتى كنترل علائم فيزيولوريك قبل از

2. Benson relaxation

3. Angina Pectoris 


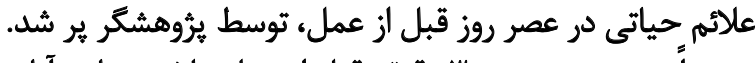

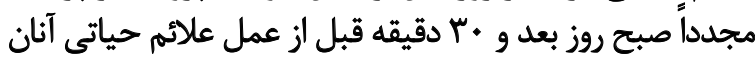

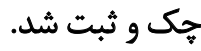
آناليز آمارى دادها

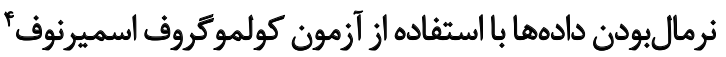

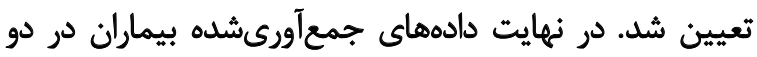

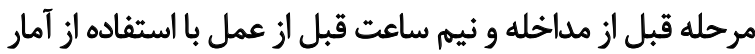

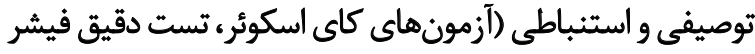

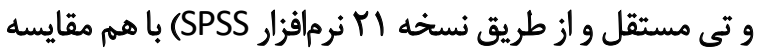

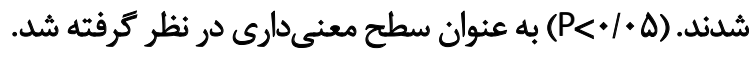

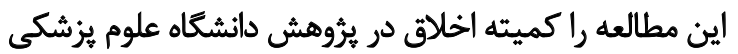

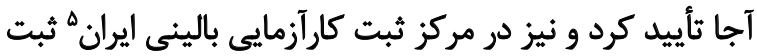

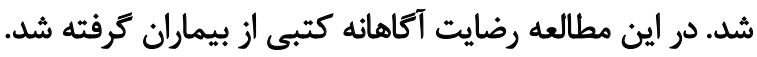

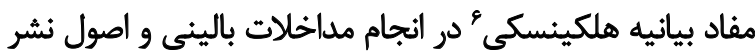
كميته اخلاق نشر بنيز رعايت شد.

يافتهها

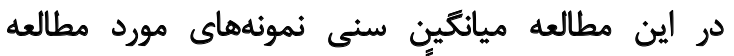

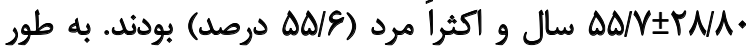

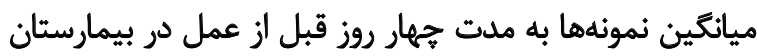

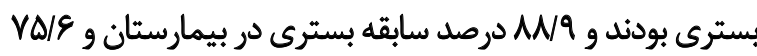
درصد سابقه جراحى داشتند.

نتايج يافتههاى توصيفى اين مطالعه نشان داد ميان ميانكين

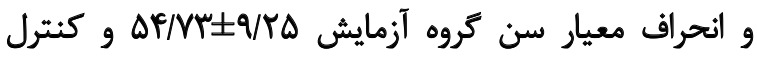

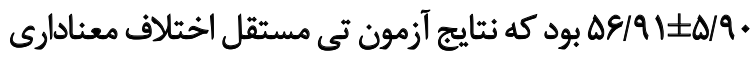

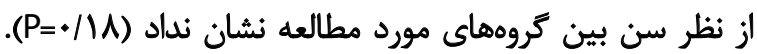

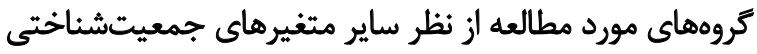

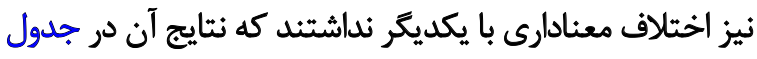

$$
\text { شماره ا نشان داده شده است. }
$$

نتايج آزمون تى مستقل نشان داد در مرحله قبل از مداخله،

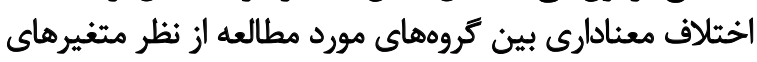

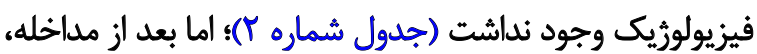

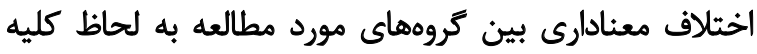

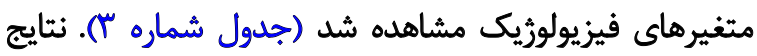

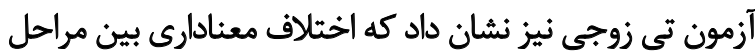

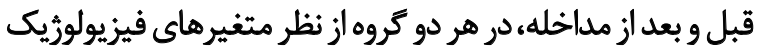

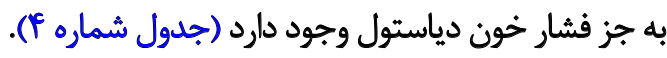

4. Kolmogorov-Smirnov test

5. IRCT

6. Declaration of helsinki

7. COPE
يُروهش استفاده شد. نبض و تعداد تنفس نيز با استفاده از ساعت

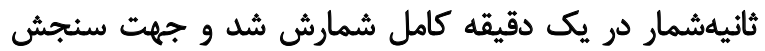

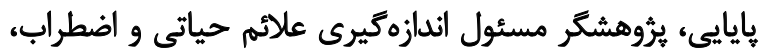

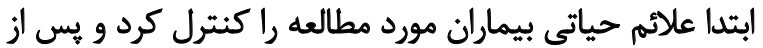

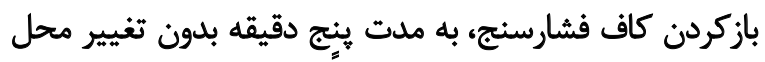

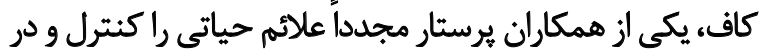

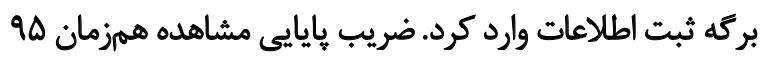
درصد محاسبه شد.

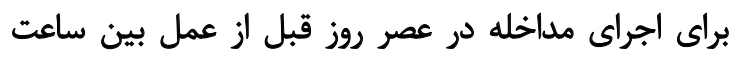

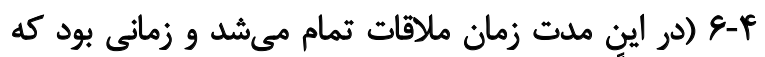

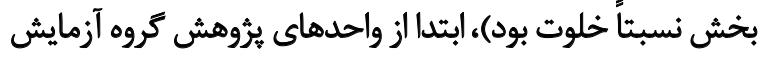

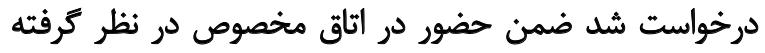

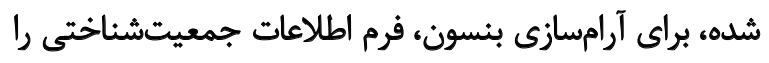

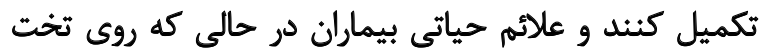

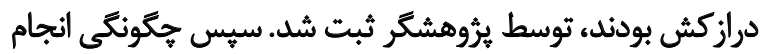

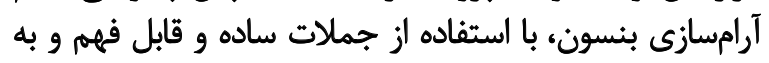

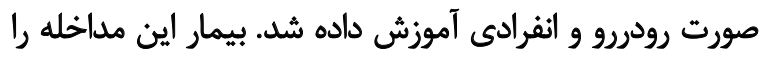

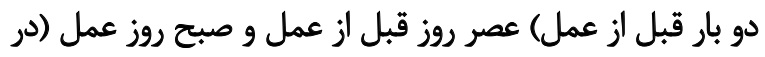

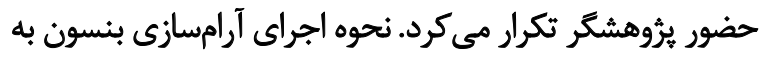
اين صورت بود: قراركيرى بيمار در بهترين وضعيتى كه در آن احساس آرامش مي كند.

رشمان بسته، انجام تنفس هاى عميق و منظمه به طورى كها از راه بينى دم و بازدم را الز راه دهان خارج كندان

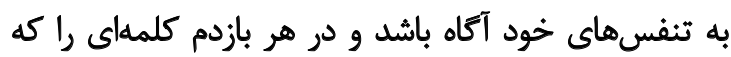

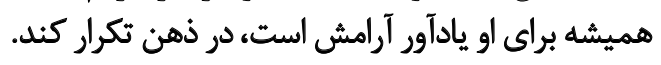

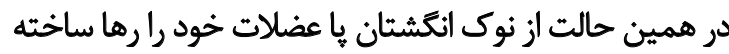

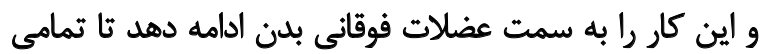
عضلات به انبساط كامل برسند. بيمار اين حالت را به مدت ·r دقيقه حفظ كند. بس از اتمام كار جئد دقيقه در همين حالت باقى بماند.

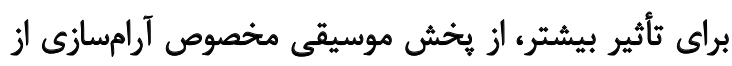

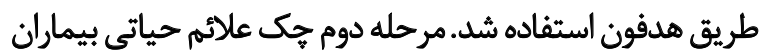

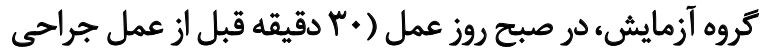

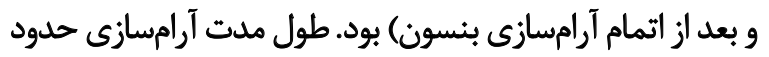

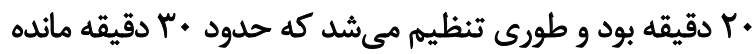

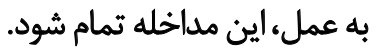

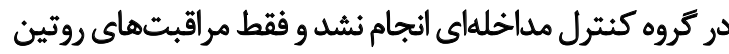

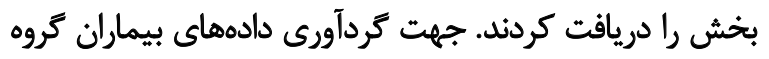

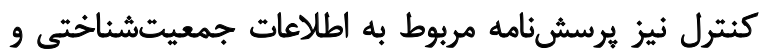


جدول ا. مقايسه متغيرهاى جمعيتشناختى كروهاي مورد مطالعه

\begin{tabular}{|c|c|c|c|c|}
\hline \multirow{2}{*}{ ت تئايج آزمون } & \multicolumn{2}{|c|}{ تعداد (درصد) } & & \multirow{2}{*}{ متغير } \\
\hline & كنترل & آزمايش & & \\
\hline \multirow{2}{*}{$\begin{array}{c}\mathrm{P}=/ / \mathrm{rV} \\
\text { Fishers Exact Test }=1 / \mathrm{T \& V}\end{array}$} & $T(F \& M)$ & $r q(e p / p)$ & مر & \multirow[b]{2}{*}{ جنس } \\
\hline & $r e(\Delta r / M)$ & $18(T \Delta / 8)$ & نj & \\
\hline \multirow{6}{*}{$\begin{array}{c}P=\cdot N \cdot V^{*} \\
d f=\emptyset \\
\text { Value }=r / 9 \Delta \&\end{array}$} & $\Delta(11 / 1)$ & $8(1 \% / r)$ & $r$ & \multirow{6}{*}{$\begin{array}{c}\text { مدت زمان بسترى در بيمارستان } \\
\text { موروز }\end{array}$} \\
\hline & $11(f / \pi f)$ & $1 \cdot(M Y M)$ & $r$ & \\
\hline & If $(r \mid l)$ & $1 \cdot(K r / Y)$ & $r$ & \\
\hline & $11(\pi / R)$ & $10\left(\pi r / T^{m}\right)$ & $\Delta$ & \\
\hline & $r(g / V)$ & $F(N q)$ & $\varepsilon$ & \\
\hline & $1(T / T)$ & . & $\mathrm{V}$ & \\
\hline \multirow{2}{*}{$\begin{array}{c}\mathrm{P}=M^{\text {*q }} \\
\text { Fishers Exact Test=//YeV }\end{array}$} & fi $(91 /)$ & rq (N\&/V) & بلى & \multirow{2}{*}{ سابقه بسترى در بيمارسثان } \\
\hline & $F(N q)$ & $q(\mid r / T)$ & خير & \\
\hline \multirow{2}{*}{$\begin{array}{c}\mathrm{P}=\bullet / \bullet \wedge \Delta \\
\text { Fishers Exact Test }=\mathrm{I} / \mathrm{K} \mathrm{V}\end{array}$} & 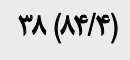 & $r \cdot(\operatorname{sg} N)$ & بلى & \multirow[b]{2}{*}{ سابقه جراحى } \\
\hline & $V(\mid \triangle / 8)$ & $10(M T / M)$ & خير & \\
\hline
\end{tabular}

$$
\text { مطالعه قدرت تعميميذيرى بيشترى ناريه بارند. }
$$

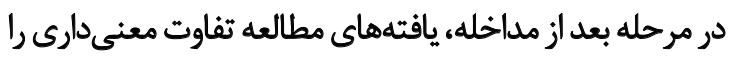

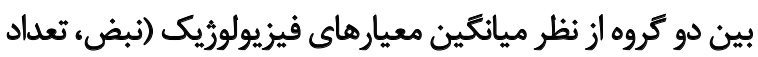
تنفس، فشار خون سيستول، فشار خون دياستول) در مرحله يس فيس نيس

اين مطالعه باهدف بررسى تأثير آرامسازى بنسون بر معيارهاى

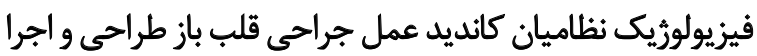

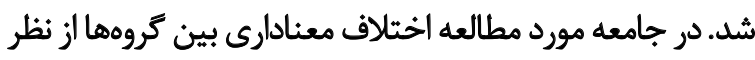

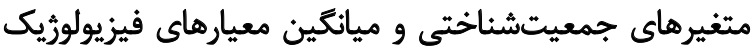
جدول r. مقايسه ميانكين متغير هاي هموديناميك در دو كروه آزمايش وكتبرل در مرحله قبل از مداخله

\begin{tabular}{|c|c|c|c|}
\hline \multirow{2}{*}{ تتيجه آزمون تى مستقل } & \multicolumn{2}{|c|}{ ميانئين } & \multirow{2}{*}{ مثغير } \\
\hline & كتئرل & آزمايش & \\
\hline $\begin{array}{c}t=q \varepsilon \Delta / \\
d f=M \\
P=m V / .\end{array}$ & $W / r+ \pm 1+/ 8$ & VQ/eV $\pm 1 r / F e$ & تعلداد نبض \\
\hline $\begin{array}{c}t=V / F / \Delta \\
d f=M \\
P=\star \varphi_{\star} / .\end{array}$ & $|9 / \Delta| \pm 1 / A C$ & $r Y / P \pm Y / Y M$ & تعلعاد تنفس \\
\hline $\begin{array}{c}t=\Delta r r / r \\
d f=M \\
P=\diamond \Delta Y /\end{array}$ & $|r \cdot / \Delta \& \pm| \nabla / \Delta T$ & $\mid r / M+18 / \Delta$. & فشار خون سيستول \\
\hline $\begin{array}{l}t=-r \mid q / . \\
d f=r \cdot|\varphi| \\
P=V \Delta Y / .\end{array}$ & $A \cdot / \Delta \& \pm T M / F \Delta$ & $v 2 / r r \pm 1 . / 01$ & فُشار خون دياستول \\
\hline
\end{tabular}


جدول ץ. مقايسه ميائكين متغير هاى هموديناميك در دو كروه آزمايش و كنترل در مرحله بعد از مداخله

\begin{tabular}{|c|c|c|c|}
\hline \multirow{2}{*}{ تتيجه أزمون تى مستقل } & \multicolumn{2}{|c|}{ ميائكين |نحراف معيار } & \multirow{2}{*}{ متغير } \\
\hline & كتيترل & أزمون & \\
\hline $\begin{array}{c}t=-r r g / \varepsilon \\
d f=M \\
P=-11 \cdots\end{array}$ & Wer $\pm 1 r / 19$ & $V T / T V \pm 11 / T q$ & تعداد ئبض \\
\hline $\begin{array}{c}t=-r \Delta) / \\
d f=M \\
P=\cdot / \cdots\end{array}$ & $r \cdot / \cdot r \pm T / M I$ & WAP $\pm N / M^{2}$ & تعلداد تنقس \\
\hline $\begin{array}{c}t=-e)^{\prime} / r \\
d f=M \\
P=.11 \cdots\end{array}$ & IrNVA $\pm r+/ T r$ & ITE/VA $\pm I \Delta / W$ & فشار خون سيستول \\
\hline $\begin{array}{c}t=-+9 \mathrm{r} / \mathrm{r} \\
\mathrm{df}=\mathrm{M} \\
\mathrm{P}=+\mathrm{HT} /\end{array}$ & $\Lambda r / \Lambda \pm q / 1 r$ & $W / \wedge q \pm 1 \cdot / r \Delta$ & فشار خُون دياستول \\
\hline
\end{tabular}

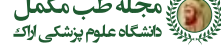

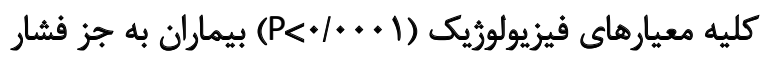

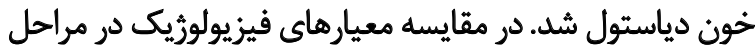

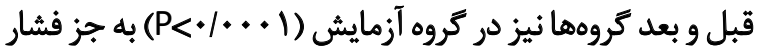

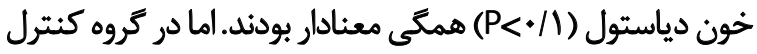

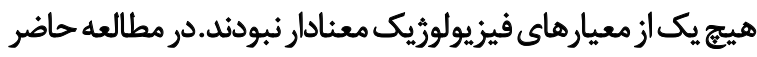

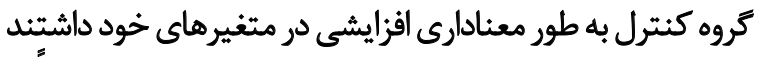

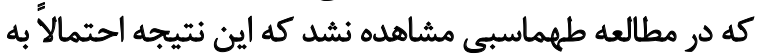

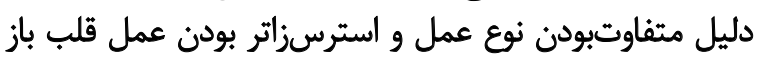

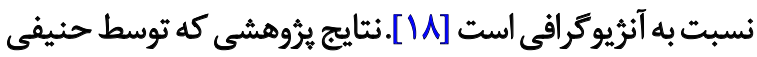

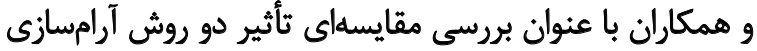

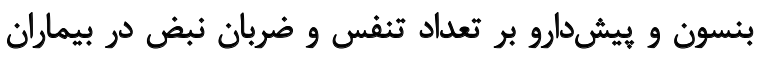

از مداخله نشان داد و نتايج حاكى از آن بود كه آرامسازى بنسون،

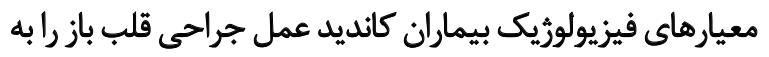

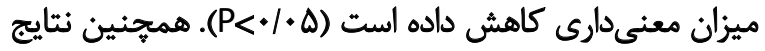

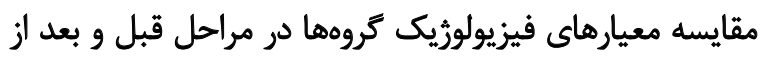

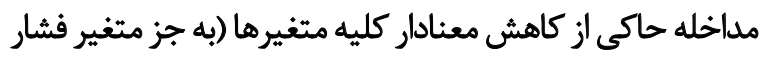

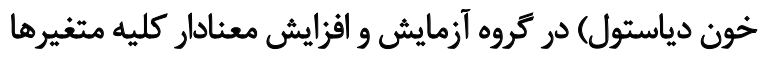

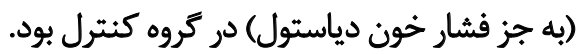

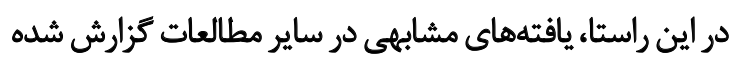

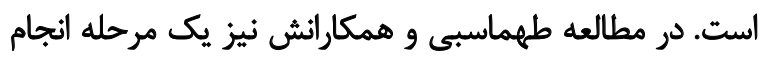

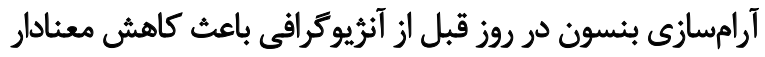

جدول †. نتايج آزمون تى زوجى در مقايسه معيارهاى هموديناميك نمونهها در مرحله قبل و بعد از مداخله در هر كروه مورد مطالعه

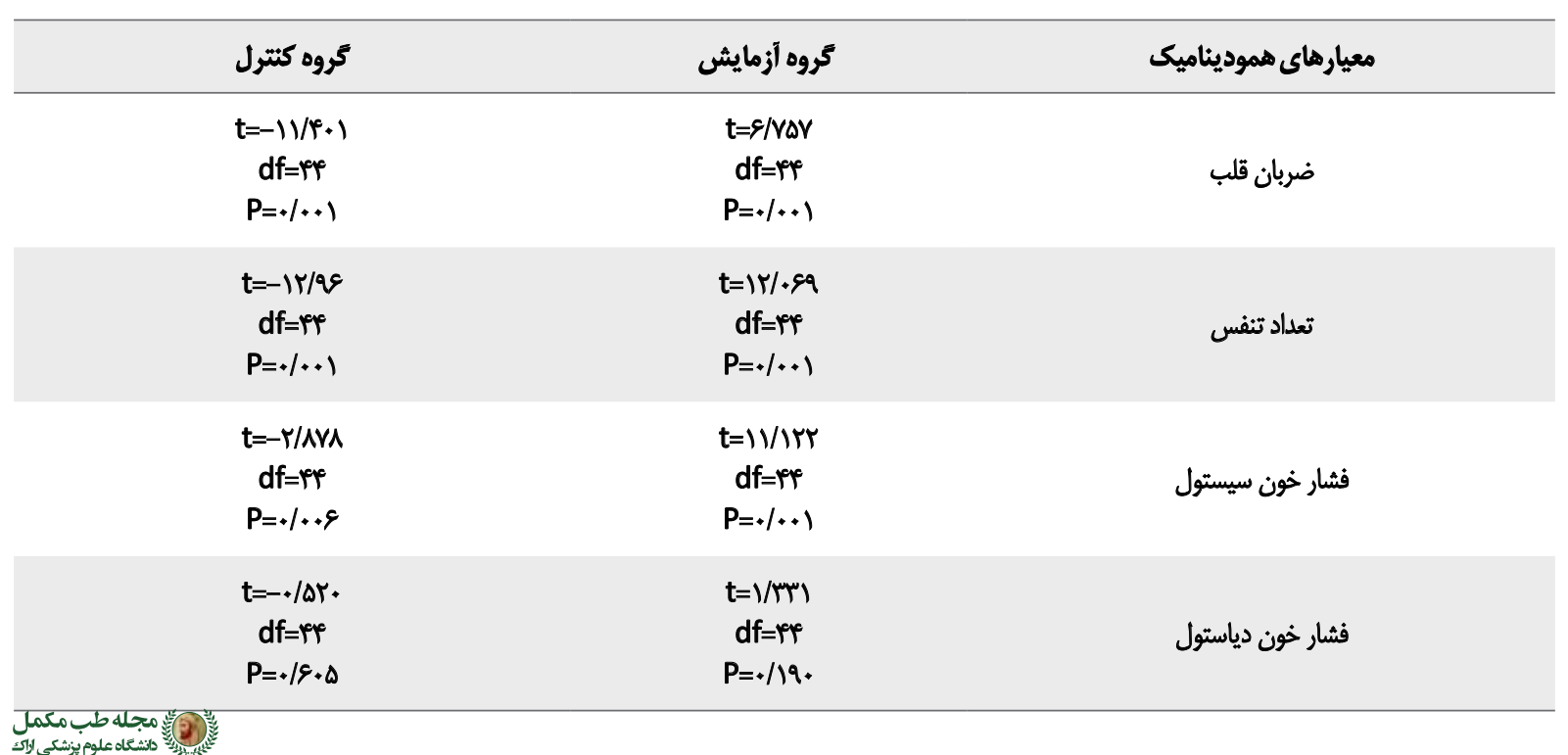


كه اين مسئله به دليل آرامشى است كه با كاهش نياز اثرزى

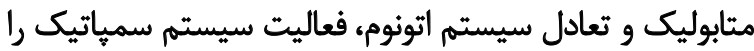

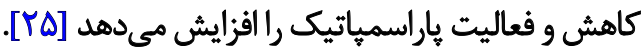

تثيجليَّى

بر اساس نتايج اين يثروهش، آرامسازى بنسون به طور معنادارى

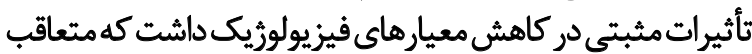

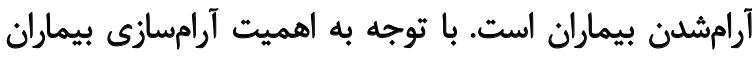

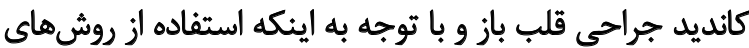

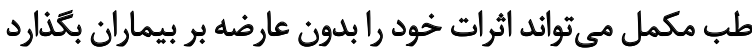

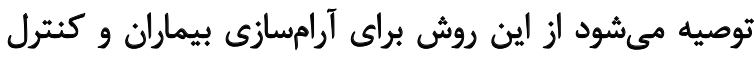

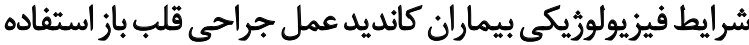

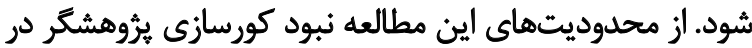

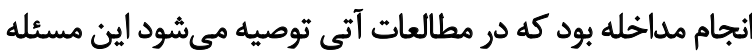

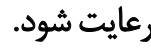

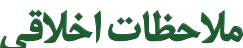

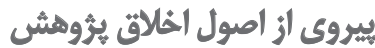

اين مطالعه را كميته اخلاق در يُوهش دانشكاه علوم يزشكى دئى

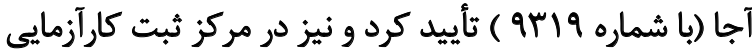
بالينى ايران (كد إنمارها

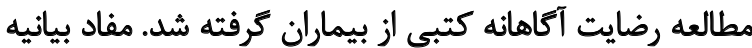
هلكينسكى در انجام مداخلات بالينى و اصول نشر كميته اخلاق

نشر نيز رعايت شد.

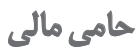

اين مطالعه حاصل يك طرح مصوب در دانشكاه علوم يزشكى

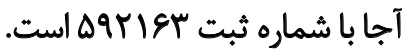

مشاركت ثويسند متًان

مفهومسازي: فاطمه تيمورى، مهلدى مالمير و سيد اميرحسين

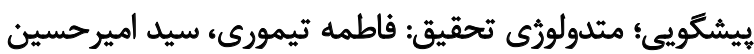

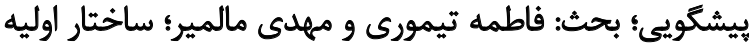

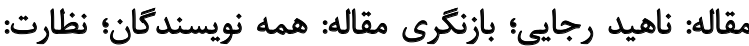

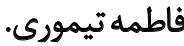

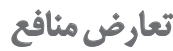

در اين مطالعه هيجَّونه تضاد منافعى وجود نداشت.

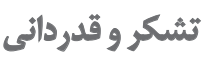

بدينوسيله از دانشكاه علوم بزشكى آجا كه حمايت مالى طرح

$$
\text { را بر عهده داشتند، تشكر و قدردانى مى دئشود. }
$$

زير آنثيوكرافى عروق كرونر انجام شد، نشان داد تعداد تنفس

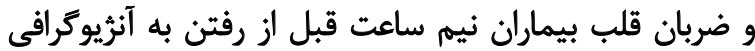

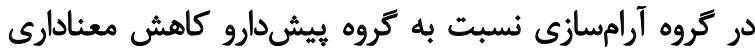

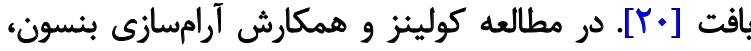

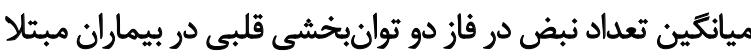

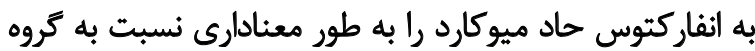

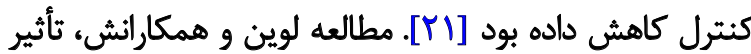

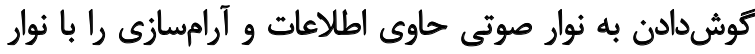

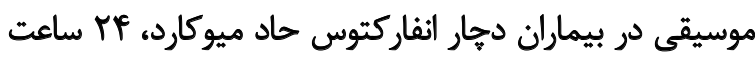

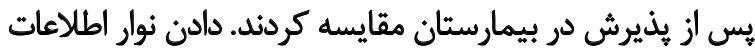

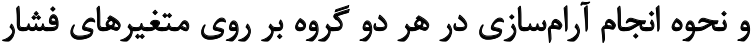

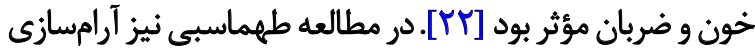

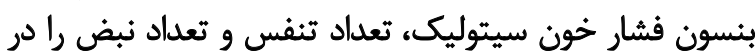

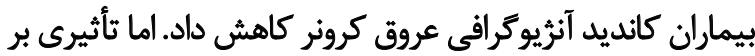
فشار خون دياستول نداشت [1/ [1]. برخلاف نتايج مطالعه حاضر، در مطالعه محمدى و همكاران،

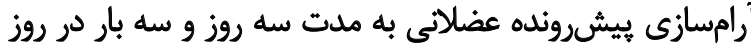

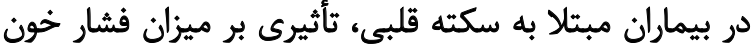

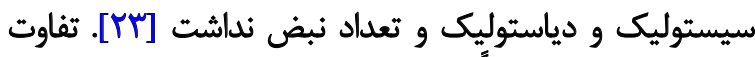

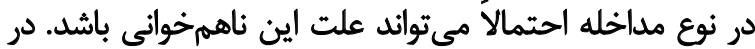

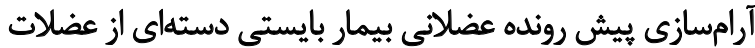

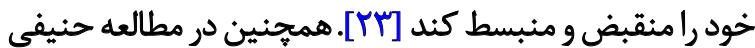

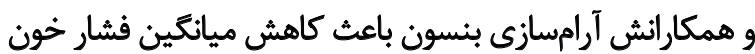

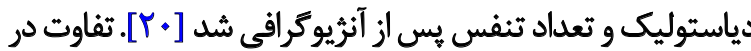

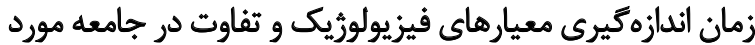
مطالعه مى تواند دليل اين اختلاف باشيد. درواقع مشاهدهنكردن تغييرات فشار خون دياستول در مقايسه

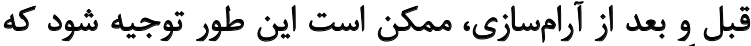

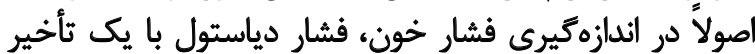

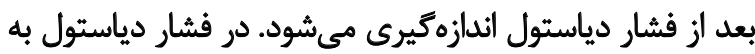

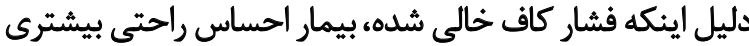

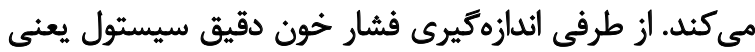

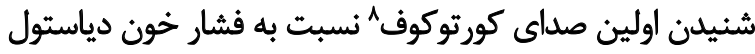

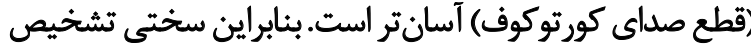
قطع صدا دقت را بايين آورده و وتغييرات فشار خون خون دياستول

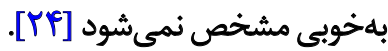

آرامسازى بئسون، باعث ايجاد تعادل بين قسمت قدامي وائي و

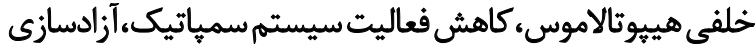

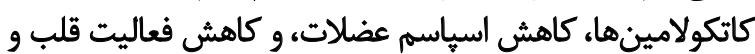

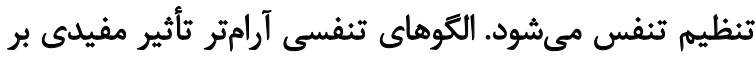

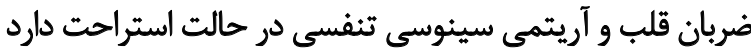




\section{References}

[1] Amiri ZS, Khajedaluee M, Rezaii A, Dadgarmoghaddam M. The risk of cardiovascular events based on the Framingham criteria in adults living in Mashhad (Iran). Electronic Physician. 2018; 10(8):7164-73. [DOI:10.19082/7164] [PMID] [PMCID]

[2] Yusuf S, Wood D, Ralston J, Srinath Reddy K. The World Heart Federation's vision for worldwide cardiovascular disease prevention. The Lancet. 2015; 386(9991):399-402. [DOI:10.1016/S0140-6736(15)60265-3]

[3] Nangia R, Singh H, Kaur K. Prevalence of Cardiovascular Disease (CVD) risk factors. Medical Journal Armed Forces India. 2016; 72(4):315-9. [DOI:10.1016/j.mjafi.2014.07.007] [PMID] [PMCID]

[4] Kustrzycki W, Rymaszewska J, Malcher K, Szczepanska-Gieracha J, Biecek P. Risk factors of depressive and anxiety symptoms 8 years after coronary artery bypass grafting. European Journal of Cardio-Thoracic Surgery. 2012; 41(2):302-6. [DOI:10.1016/j.ejcts.2011.06.028] [PMID]

[5] Akomea-Agyin C, Galukande M, Mwambu T, Ttendo S, Clarke I. Pioneer human open heart surgery using cardiopulmonary by pass in Uganda. African Health Sciences. 2008; 8(4):259-60. [PMID] [PMCID]

[6] Jalali Farahani AR, Naseri MH, Lal Dolat Abadi H, Arab Salmani I, Jonaidi Jafari NA, Teymoori M. [Comparative study of cardiovascular risk factor between military patient and non-military patient in Shahid Rajaee and Baqiyatallah Hospitals (Persian)]. Journal of Military Medicine. 2008; 10(2):137-42.

[7] Gonçalves KKN, da Silva Jl, Gomes ET, de Souza Pinheiro LL, Figueiredo TR, da Silva Bezerra SMM. [Anxiety in the preoperative period of heart surgery (English, Portuguese)]. Revista Brasileira de Enfermagem. 2016; 69(2):397-403. [DOI:10.1590/0034-7167.2016690225i] [PMID]

[8] Vingerhoets G. Perioperative anxiety and depression in open-heart surgery. Psychosomatics. 1998; 39(1):30-7. [DOI:10.1016/S00333182(98)71378-7]

[9] Tully PJ, Baker RA, Knight JL. Anxiety and depression as risk factors for mortality after coronary artery bypass surgery. Journal of Psychosomatic Research. 2008; 64(3):285-90. [DOI:10.1016/j.jpsychores.2007.09.007] [PMID]

[10] Lenzen MJ, Gamel CJ, Immink AW. Anxiety and well-being in firsttime coronary angioplasty patients and repeaters. European Journal of Cardiovascular Nursing. 2002; 1(3):195-201. [DOI:10.1016/S14745151(02)00035-X]

[11] Turton MB, Deegan T, Coulshed N. Plasma catecholamine levels and cardiac rhythm before and after cardiac catheterization. British Heart Journal. 1977; 39(12):1307-11. [DOI:10.1136/hrt.39.12.1307] [PMID] [PMCID]

[12] Gunnarsdottir TJ, Jonsdottir H. Does the experimental design capture the effects of complementary therapy? A study using reflexology for patients undergoing coronary artery bypass graft surgery. Journal of Clinical Nursing. 2007; 16(4):777-85. [DOI:10.1111/j.13652702.2006.01634.x] [PMID]

[13] Rahmani Anaraki H, Abdollahi AA, Nasiri H, Vakili MA. [Immediate effects of a five minutes back massage on patients' physiological parameters in critical care unit (Persian)]. Journal of Gorgan University of Medical Sciences. 2001; 3(2):53-8.

[14] Pudner R, editor. Nursing the surgical patient. 2nd ed. Philadelphia: Elsevier; 2005.

[15] Benson H, Kotch JB, Crassweller KD. The relaxation response: A bridge between psychiatry and medicine. Medical Clinics of North America. 1997; 61(4):929-38. [DOI:10.1016/S0025-7125(16)31308-6]
[16] van Dixhoorn J, White A. Relaxation therapy for rehabilitation and prevention in ischaemic heart disease: A systematic review and meta-analysis. European Journal of Preventive Cardiology. 2005; 12(3):193-202. [DOI:10.1097/01.hjr.0000166451.38593.de] [PMID]

[17] Torabi M, Salavati M, Ghahri Sarabi AR, Pouresmaiel Z, Akbarian Baghban AR. [Effect of foot reflexology massage and Benson relaxation techniques on anxiety and physiological indexes of patients undergoing coronary heart angiography (Persian)]. Avicenna Journal of Nursing and Midwifery Care. 2012; 20(1):63-71.

[18] Tahmasbi H, Hasani S. Effect of Benson's relaxation technique on the anxiety of patients undergoing coronary angiography: A randomized control trial. Journal of Nursing and Midwifery Sciences. 2016; 3(1):814. [DOI:10.18869/acadpub.jnms.3.1.8]

[19] Hazraty M, Hoseini M, Dejbakhsh T, Taghavi A, Rajaee-fard A. [The effect of Benson relaxation therapy on anxiety level and severity of symptoms in patients with irritable bowel syndrome (Persian)]. Journal of Arak University of Medical Sciences. 2006; 9(4):1-9.

[20] Hanifi N, Ahmadi F, Memarian R, Khani M. [Effect of Benson relaxation techniques on hemodynamic variables of patient undergoing coronary angiography (Persian)]. Journal of Shahid Sadoughi University of Medical Sciences. 2005; 12(4):78-86.

[21] Collins JA, Rice VH. Effects of relaxation intervention in phase II cardiac rehabilitation: Replication and extension. Heart \& Lung. 1997; 26(1):3144. [DOI:10.1016/S0147-9563(97)90007-8]

[22] Lewin RJP, Thompson DR, Elton RA. Trial of the effects of an advice and relaxation tape given within the first $24 \mathrm{~h}$ of admission to hospital with acute myocardial infarction. International Journal of Cardiology. 2002; 82(2):107-14. [DOI:10.1016/S0167-5273(01)00620-9]

[23] Mohamadi F, Ahmadi F, Nematipour E, Faghihzadh S. [The effect of progressive muscle relaxation method on vital signs in myocardial infraction patients (Persian)]. Koomesh. 2006; 7(3-4):189-95. [Persian]

[24] Musini VM, Wright JM. Factors affecting blood pressure variability: Lessons learned from two systematic reviews of randomized controlled trials. PLoS One. 2009; 4(5):e5673. [DOI:10.1371/journal.pone.0005673] [PMID] [PMCID]

[25] van Dixhoorn J. Cardiorespiratory effects of breathing and relaxation instruction in myocardial infarction patients. Biological Psychology. 1998; 49(1-2):123-35. [DOI:10.1016/S0301-0511(98)00031-3] 